OPEN ACCESS

Edited by:

Ruiwen Zhang,

Texas Tech University Health

Sciences Center, USA

Reviewed by:

Jianwei Zhou,

Nanjing Medical University, China

Hongxia Xu,

Third Military Medical University,

China

*Correspondence:

Zhenzhou Jiang

beaglejiang@cpu.edu.cn

Luyong Zhang

lyzhang@cpu.edu.cn

Huiping Zhou

huiping.zhou@vcuhealth.org

Specialty section: This article was submitted to

Ethnopharmacology,

a section of the journal

Frontiers in Pharmacology

Received: 17 October 2016 Accepted: 25 April 2017

Published: 09 May 2017

Citation:

Yang J, Sun L, Wang L, Hassan HM, Wang $X$, Hylemon PB, Wang T,

Zhou H, Zhang L and Jiang Z (2017) Activation of Sirt1/FXR Signaling Pathway Attenuates

Triptolide-Induced Hepatotoxicity in Rats. Front. Pharmacol. 8:260. doi: 10.3389/fphar.2017.00260

\section{Activation of Sirt1/FXR Signaling Pathway Attenuates Triptolide-Induced Hepatotoxicity in Rats}

\author{
Jing Yang 1,2, Lixin Sun 1, Lu Wang ${ }^{1}$, Hozeifa M. Hassan 1, Xuan Wang ${ }^{2}$, \\ Phillip B. Hylemon ${ }^{2,3}$, Tao Wang ${ }^{1}$, Huiping Zhou ${ }^{2,3 *}$, Luyong Zhang ${ }^{1,4,5 *}$ and \\ Zhenzhou Jiang ${ }^{1,6,7 *}$
}

1 Jiangsu Key Laboratory of Drug Screening, China Pharmaceutical University, Nanjing, China, ${ }^{2}$ Department of Microbiology and Immunology, Virginia Commonwealth University, Richmond, VA, USA, ${ }^{3}$ McGuire Veterans Affairs Medical Center, Richmond, VA, USA, ${ }^{4}$ Jiangsu Center for Pharmacodynamics Research and Evaluation, China Pharmaceutical University, Nanjing, China, ${ }^{5}$ State Key Laboratory of Natural Medicines, China Pharmaceutical University, Nanjing, China, ${ }^{6}$ Key Laboratory of Drug Quality Control and Pharmacovigilance, China Pharmaceutical University, Ministry of Education, Nanjing, China, ${ }^{7}$ Jiangsu Key Laboratory of TCM Evaluation and Translational Research, China Pharmaceutical University, Nanjing, China

Triptolide (TP), a diterpenoid isolated from Tripterygium wilfordii Hook F, has an excellent pharmacological profile of immunosuppression and anti-tumor activities, but its clinical applications are severely restricted due to its severe and cumulative toxicities. The farnesoid $X$ receptor $(F X R)$ is the master bile acid nuclear receptor and plays an important role in maintaining hepatic metabolism homeostasis. Hepatic Sirtuin (Sirt1) is a key regulator of the FXR signaling pathway and hepatic metabolism homeostasis. The aims of this study were to determine whether Sirt1/FXR signaling pathway plays a critical role in TP-induced hepatotoxicity. Our study revealed that the intragastric administration of TP (400 $\mu \mathrm{g} / \mathrm{kg}$ body weight) for 28 consecutive days increased bile acid accumulation, suppressed hepatic gluconeogenesis in rats. The expression of bile acid transporter BSEP was significantly reduced and cholesterol $7 \alpha$-hydroxylase (CYP7A1) was markedly increased in the TP-treated group, whereas the genes responsible for hepatic gluconeogenesis were suppressed in the TP-treated group. TP also modulated the FXR and Sirt1 by decreasing its expression both in vitro and in vivo. The Sirt1 agonist SRT1720 and the FXR agonist obeticholic acid (OCA) were used both in vivo and in vitro. The remarkable liver damage induced by TP was attenuated by treatment with either SRT1720 or OCA, as reflected by decreased levels of serum total bile acids and alkaline phosphatase and increased glucose levels. Meanwhile, SRT1720 significantly alleviated TP-induced FXR suppression and FXR-targets involved in hepatic lipid and glucose metabolism. Based on these results, we conclude that Sirt1/FXR inactivation plays a critical role in TP-induced hepatotoxicity. Moreover, Sirt1/FXR axis represents a novel therapeutic target that could potentially ameliorate TP-induced hepatotoxicity.

Keywords: triptolide, hepatotoxicity, bile acid metabolism, hepatic gluconeogenesis, farnesoid X receptor, Sirtuin 1 


\section{INTRODUCTION}

The diterpenoid triepoxide triptolide (TP) is the major active component that is extracted from the traditional Chinese medicine, Tripterygium wilfordii Hook F (TWHF). TP has multiple pharmacological effects, including anti-inflammatory, immunosuppressive and anti-proliferative activities (Zhou et al., 2012; Ziaei and Halaby, 2016). TP is commonly used to treat certain autoimmune and inflammatory disorders. Minnelide, a water-soluble analog of TP and a synthetic pro-drug, is being studied as a potent chemotherapeutic drug for pancreatic cancer (Rivard et al., 2014; Banerjee and Saluja, 2015). However, due to its narrow therapeutic window and severe toxicities, especially hepatotoxicity, the clinical applications of TP are limited (Zhou et al., 1999). Previous studies have indicated that the TP-induced hepatotoxicity is possibly associated with increased lipid peroxidation (Jiang et al., 2016), DNA damage, mitochondrial impairment (Yao et al., 2008; Fu et al., 2011, 2013) or Th17/Treg imbalance (Wang et al., 2014). However, the exact cellular/molecular mechanisms by which TP induces hepatotoxicity remain unclear. There is an urgent need to develop novel therapeutic strategies to prevent or counteract the TPinduced hepatotoxicity.

The farnesoid X receptor (FXR) is the master nuclear receptor for bile acids and plays an important role in maintaining hepatic bile acid homeostasis through activation of the small heterodimer partner (SHP), which represses the expression of CYP7A1 (Noel et al., 2016). Additionally, FXR also positively regulates essential bile acid transporters such as the ATP-binding cassette, sub-family B member 11 (ABCB11; BSEP) (Chen et al., 2016). Moreover, FXR is involved not only in the regulation of bile acid metabolism, but also in the modulation of glucose metabolism (Stayrook et al., 2005; Watanabe et al., 2006). FXR agonists or bile acids induced a rapid down-regulation of the rate-limiting gluconeogenic genes PEP carboxykinase (PEPCK) and glucose-6-phophatase (G6PC) in the liver (Stayrook et al., 2005; Cao et al., 2010). In addition, studies from several laboratories have shown that FXR is a possible target protein that mediates drug-induced liver injury (DILI) and FXR antagonism may help in the development of DILI (Cariou, 2008). Furthermore, activation of FXR by its agonist GW4064 or by obeticholic acid (OCA) attenuated some forms of DILI (Liu et al., 2003; Mudaliar et al., 2013; Jin et al., 2015). However, activation of AMP-activated protein kinase (AMPK) directly phosphorylates FXR and inhibits its activation and subsequently down-regulates the expression of FXR-targeted genes, which is associated with liver injury under cholestatic conditions (Lien et al., 2014). Consequently, reactivating FXR may be a potential therapeutic strategy for the treatment of DILI.

Sirtuin 1 (Sirt1), a class III NAD+-dependent histone deacetylase, is involved in lipid, glucose and bile acid metabolism. Because it lacks a DNA-binding domain, Sirt1 is recruited to target promoters by sequence-specific transcription factors like FXR. Deacetylation of the transcription factors by Sirt1 results in alterations in gene transcription. Not surprisingly, Sirt1 modulates the FXR-stimulated transcriptional signaling by deacetylation of this nuclear receptor and neighboring histones that strictly control the target gene transcription (Kemper et al., 2009; Garcia-Rodriguez et al., 2014). Accumulating evidence suggests that activation of AMPK leads to increased cellular NAD+ levels, which subsequently modulates the Sirtl signaling (Canto et al., 2009).

In this study, we tested the hypothesis that TP suppresses the FXR pathway by reducing the Sirt1 activity, which leads to the dysregulation of the key genes involved in hepatic metabolism including SHP, BSEP, PEPCK, G6PC, and CYP7A1 and eventually induces liver injury. These results identified a potential mechanism associated with TP-induced liver injury and potential targets that could be applied to prevent or ameliorate TP-induced liver injury.

\section{MATERIALS AND METHODS}

\section{Materials}

Triptolide ( $>98 \%$, HPLC) was a gift from the Institute of Dermatology, Chinese Academy of Medical Sciences and Peking Union Medical College (Nanjing, China). GW4064 was purchased from Sigma Chemical Co. (St. Louis, MO, USA). OCA was obtained from Shanghai Houpu Chemical Co. (Shanghai, China). SRT1720 was purchased from Shanghai Yongcan Chemical Co. (Shanghai, China). RIPA lysis buffer was purchased from Beyotime (Nanjing, China). The Bio-Rad protein assay reagent, Precision Plus Protein Kaleidoscope Standards, $4 \times$ sample buffer and SYBR Green Supermix were from Bio-Rad (Hercules, CA, USA). The primary antibodies against FXR (sc13063), SHP (sc-30169), CYP7A1 (sc-25536), PEPCK (sc-32879), and $\beta$-actin (sc-47778) were from Santa Cruz Biotechnology (Santa Cruz, CA, USA). The phospho-AMPK (2535s), AMPK (2532), and Sirt1 (9475s) primary antibodies were from Cell Signaling Technology (Danvers, MA, USA).

\section{Isolation and Culture of Primary Rat Hepatocytes}

Primary rat hepatocytes were prepared from female Wister rats and seeded in collagen-coated $60 \mathrm{~mm}$ dish at $2 \times 10^{6}$ cells per dish as previously described (Studer et al., 2012). The cells were cultured in serum-free Williams' E medium containing dexamethasone $(0.1 \mu \mathrm{M})$, penicillin (100 units/ml), and thyroxine $(1 \mu \mathrm{M})$.

\section{Animal Studies}

Wistar rats (female, 180-200 g) were purchased from the SLRC Laboratory Animal Company (Shanghai, China). All experiments and procedures involving rats were approved by the Ethical Committee of China Pharmaceutical University, and the Laboratory Animal Management Committee of Jiangsu Province (Approval No.: 2110748) and were conducted in accordance with all applicable regulations.

The rats were raised in an aseptic facility (temperature: $24 \pm 2{ }^{\circ} \mathrm{C}$, relative humidity: $40 \pm 10 \%$ ) with 12 -h light/dark cycle and given free access to food and water. The animals 
were acclimated for one week before the experiments. The rats were randomly divided into four groups $(n=6$ /group): (1) control; (2) TP (400 $\mu \mathrm{g} / \mathrm{kg} /$ day); (3) OCA (15 mg/kg/day) +TP (400 $\mu \mathrm{g} / \mathrm{kg} /$ day); and (4) SRT1720 (10 mg/kg/day) +TP
(400 $\mu \mathrm{g} / \mathrm{kg} /$ day). The rats were fed a standard rat chow and gavaged daily with control solution $(0.5 \% \mathrm{CMC}-\mathrm{Na})$, or TP for 4 weeks. Then, all rats were fasted for $12 \mathrm{~h}$, and blood samples and liver tissues were collected for analysis.
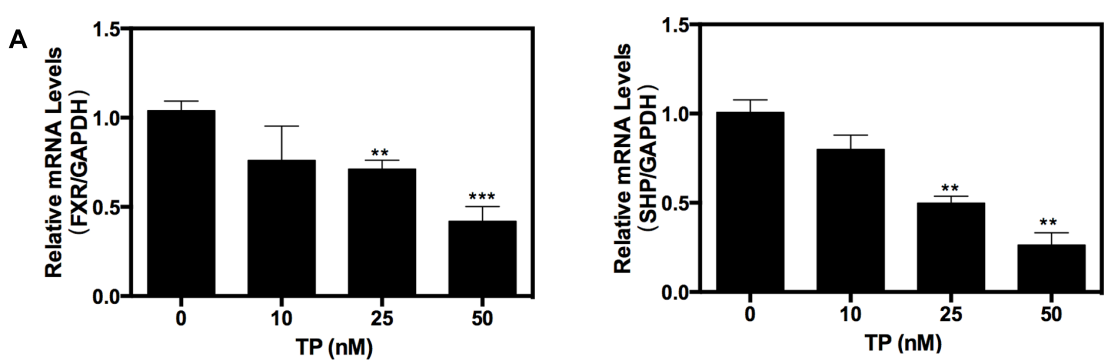

B
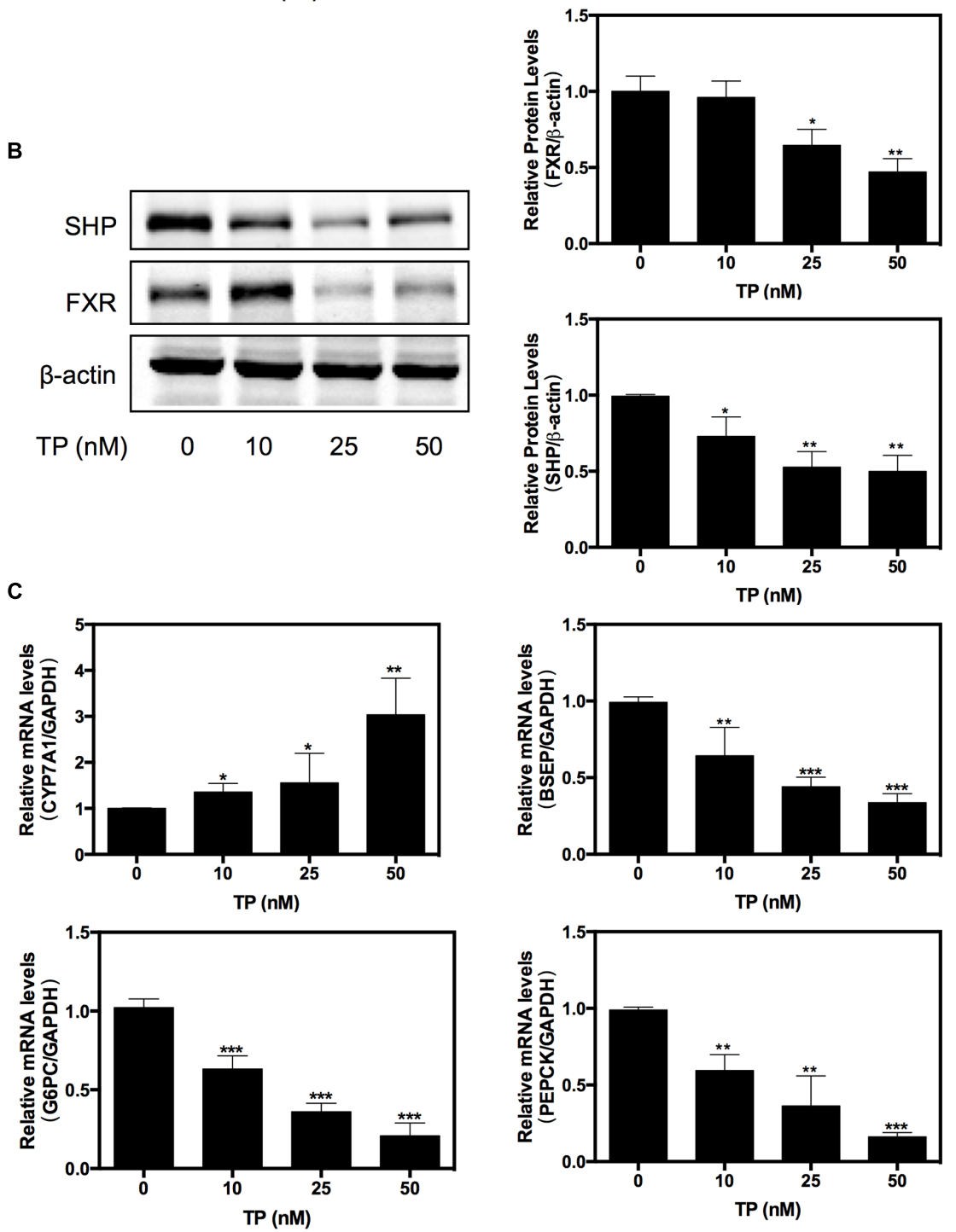

FIGURE 1 | Triptolide (TP) inhibits farnesoid X receptor (FXR) pathway in primary rat hepatocytes. Primary rat hepatocytes were treated with TP at various concentrations ( $0-50 \mathrm{nM}$ ) for $8 \mathrm{~h}$. (A) The mRNA level of FXR was measured using real-time RT-PCR and normalized to GAPDH as an internal control. (B) The protein expression level of FXR was determined using western blot analysis and normalized to $\beta$-actin as a loading control. (C) The mRNA levels of FXR-target genes were measured using real-time RT-PCR and normalized to GAPDH as an internal control. Statistical significance relative to vehicle control: ${ }^{*} p<0.05,{ }^{* *} p<0.01$, *** $p<0.001$ 


\section{RNA Extraction and Real-Time PCR}

The total RNA was isolated from the liver tissues or primary hepatocytes using TRIzol Reagent (Carlsbad, CA, USA) and reversed transcribed into cDNA using the High-capacity cDNA Reverse Transcription Kit (Waltham, MA, USA). The mRNA levels of FXR, SHP, CYP7A1, BSEP, PEPCK, G6PC, and Sirt1 were determined using real-time PCR with Thermo Hygreen Supermix reagents and normalized to GAPDH as the internal control. The primer sequences used were listed in Supplementary Table S1.

\section{Western Blot Analysis}

The total cellular proteins were collected by lysis with RIPA lysis buffer. The protein concentration was determined using the BioRad protein assay reagent. The total proteins $(100 \mu \mathrm{g})$ were resolved on $10 \%$ SDS-PAGE gel and transferred to nitrocellulose membranes. The immunoreactive bands were detected using horseradish peroxidase-conjugated secondary antibodies and ECL reagents. The bands were analyzed using Quantity One.

\section{Statistical Analysis}

The data are expressed as the means \pm SEM. One-way analysis of variance (ANOVA) and Dunnett's $t$-test was used to evaluate the differences among the various groups using GraphPad Prism 5.0 (San Diego, CA, USA). The differences were considered statistically significant at $p<0.05$.

\section{RESULTS}

\section{TP Inhibited FXR Pathway in Primary Rat Hepatocytes}

Farnesoid X receptor is an important regulator of hepatic metabolism. We investigated whether TP had any effect on

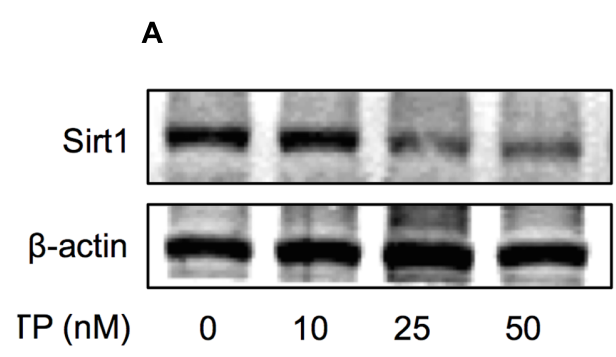

B
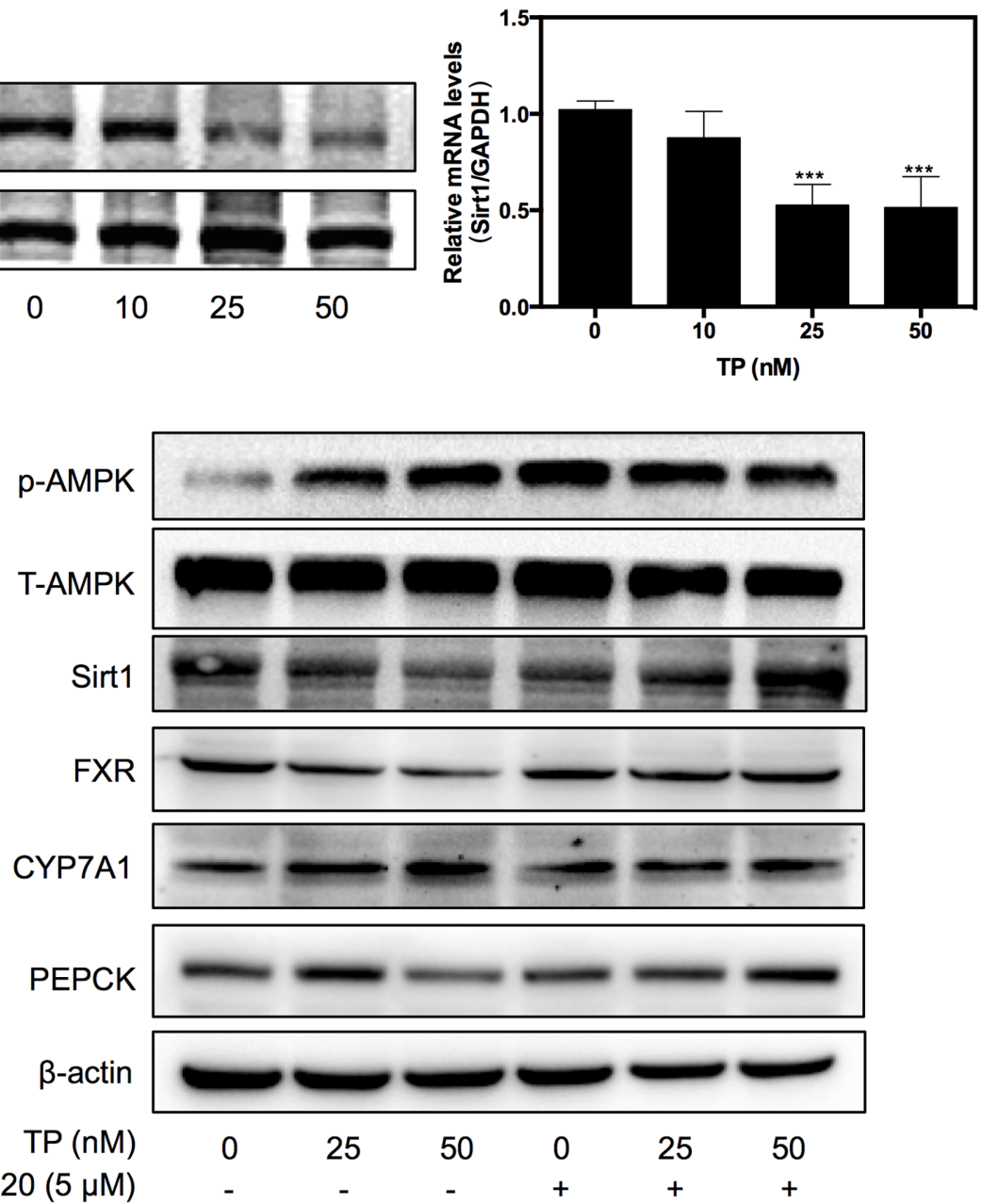

FIGURE 2 | Effect of Sirtuin (Sirt1) in TP-induced FXR suppression. (A) Primary rat hepatocytes were treated with TP for $8 \mathrm{~h}$. Sirt1 expression was determined using real-time RT-PCR and western blot analysis. (B) Primary rat hepatocytes were pretreated with a Sirt1 agonist, SRT1720 (5 $\mu$ M), for one hour and then treated with TP for $8 \mathrm{~h}$. The specific protein levels were determined using western blot analysis and normalized to $\beta$-actin as a loading control. Statistical significance relative to vehicle control, ${ }^{*} p<0.05,{ }^{* *} p<0.01,{ }^{* * *} p<0.001$; and statistical significance relative to the corresponding treatment group without SRT1720, ${ }^{\#} p<0.05$, $\# p<0.01, \# \# p<0.001$. 
hepatic FXR expression. Primary rat hepatocytes were treated with various concentrations of TP $(0,10,25,50 \mathrm{nM})$ for $8 \mathrm{~h}$. As shown in Figures $\mathbf{1 A - B}$, at 25 or $50 \mathrm{nM}$ concentration, TP dramatically inhibited expression of FXR and SHP at both the mRNA and protein levels in primary rat hepatocytes. Since FXR plays a critical role in regulating bile acid and glucose metabolism, we further examined the expression of the key genes involved in bile acid synthesis and transport as well as hepatic gluconeogenesis. The results shown in Figure 1C indicated that TP dose-dependently up-regulated the mRNA expression of CYP7A1, but it suppressed the mRNA expression of BSEP, PEPCK, and G6PC. These results suggest that inhibition of FXR expression may contribute to TP-induced dysregulation of hepatic metabolism and hepatotoxicity.

\section{TP Induced FXR Suppression via Sirt1 In Vitro}

Sirtl is a NAD+-dependent deacetylase, which deacetylates histone and non-histone proteins such as FXR. To identify the upstream signaling pathway involved in TP-induced FXR inactivation, the effect of TP on Sirt1 expression was examined. As shown in Figure 2A, TP suppressed Sirt1 expression at both the mRNA and protein levels.

Based on the observations that TP suppressed both FXR and Sirt1 expression, we used a synthetic Sirt1 agonist, SRT1720, to investigate whether activation of Sirt1 could have a protective effect against TP-induced liver injury. As shown in Figure 2B, SRT1720 (5 $\mu \mathrm{M})$ significantly alleviated TP-induced downregulation of FXR protein expression, but had no effect on TP-induced AMPK activation. To further assess whether activation of Sirtl also prevents TP-induced dysregulation of the expression of key genes involved in hepatic lipid and glucose metabolism, primary rat hepatocytes were pretreated with SRT1720 and then treated with TP. The protein levels of the FXR-targets, CYP7A1 and PEPCK, were measured using western blotting. As shown in Figure 2B, CYP7A1, a ratelimiting enzyme of bile acid synthesis, was markedly increased by TP and this effect was reduced by pretreatment with SRT1720. TP-induced inhibition of PEPCK protein expression, a key regulator of hepatic gluconeogenesis, was also blocked by SRT1720. These results indicated that Sirt1 inhibition was responsible for TP-induced FXR suppression and disruption of hepatic metabolism.

\section{Activation of FXR Ameliorates TP-Induced Hepatotoxicity}

In order to further delineate the role of FXR in TP-induced hepatotoxicity, a synthetic FXR agonist, GW4064, was used. TP-induced dysregulation of the expression of hepatic bile acid transporter BSEP and the key genes of hepatic gluconeogenesis, PEPCK and G6PC as well as CYP7A1, were significantly inhibited by GW4064 (Figure 3). These results indicated that activation of FXR could reduce TP-induced hepatotoxicity.

To further evaluate the protective role of FXR against TPinduced hepatotoxicity, primary rat hepatocytes were pretreated with OCA, which is a semi-synthetic bile acid analog and the first FXR agonist in a human clinical trial, and then treated with TP. As shown in Figure 4A, OCA partially attenuated the TP-induced dys-regulation of the mRNA expression of BSEP, CYP7A1 and SHP. Western blots further indicated that OCA prevented TPinduced down-regulation of FXR protein expression (Figure 4B). However, OCA had no effect on TP-induced down-regulation
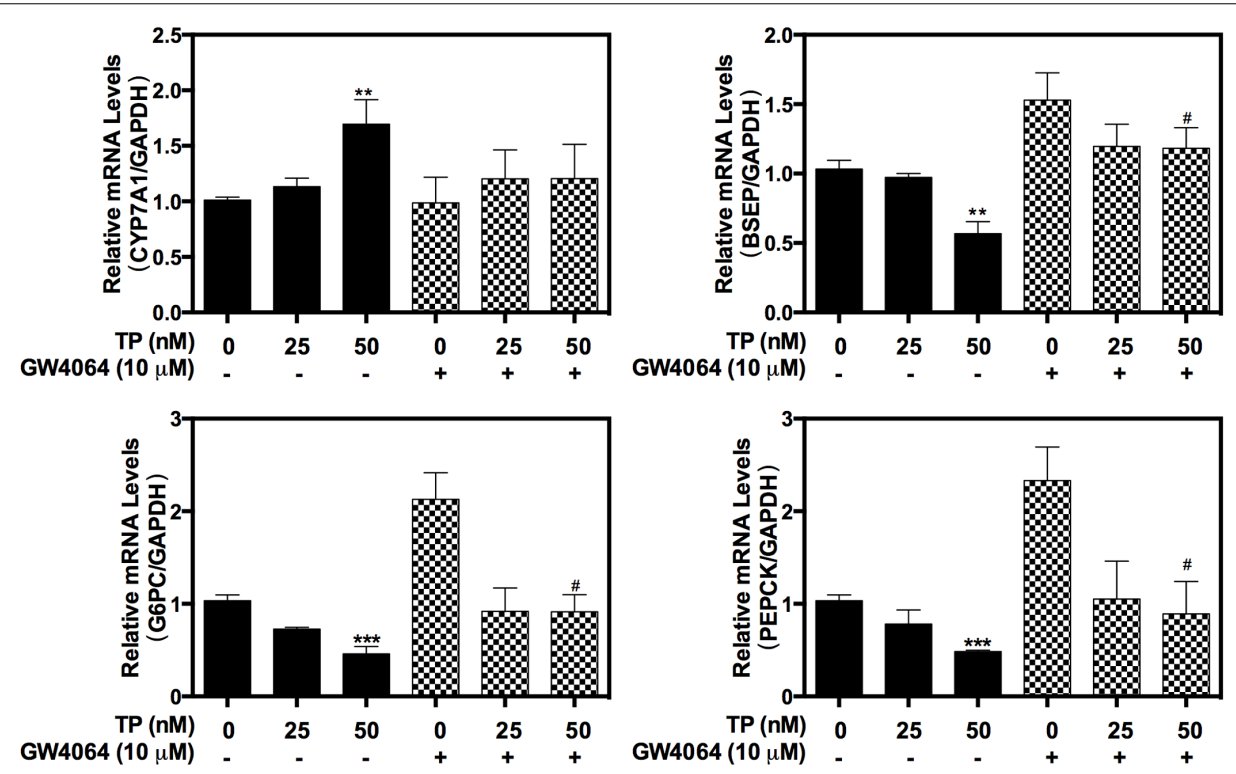

FIGURE 3 | Effect of GW4064 on TP-induced hepatotoxicity in primary rat hepatocytes. Primary rat hepatocytes were pretreated with the FXR agonist, GW4064 $(10 \mu \mathrm{M})$, for one hour and then treated with TP for $8 \mathrm{~h}$. The mRNA levels of FXR-target genes were determined using real-time RT-PCR and normalized to GAPDH as an internal control. Statistical significance relative to vehicle control, ${ }^{*} p<0.05,{ }^{* *} p<0.01$, ${ }^{* * *} p<0.001$; and statistical significance relative to the corresponding treated groups without GW4046, ${ }^{\#} p<0.05,{ }^{\# \#} p<0.01,{ }^{\# \# \#} p<0.001$. 

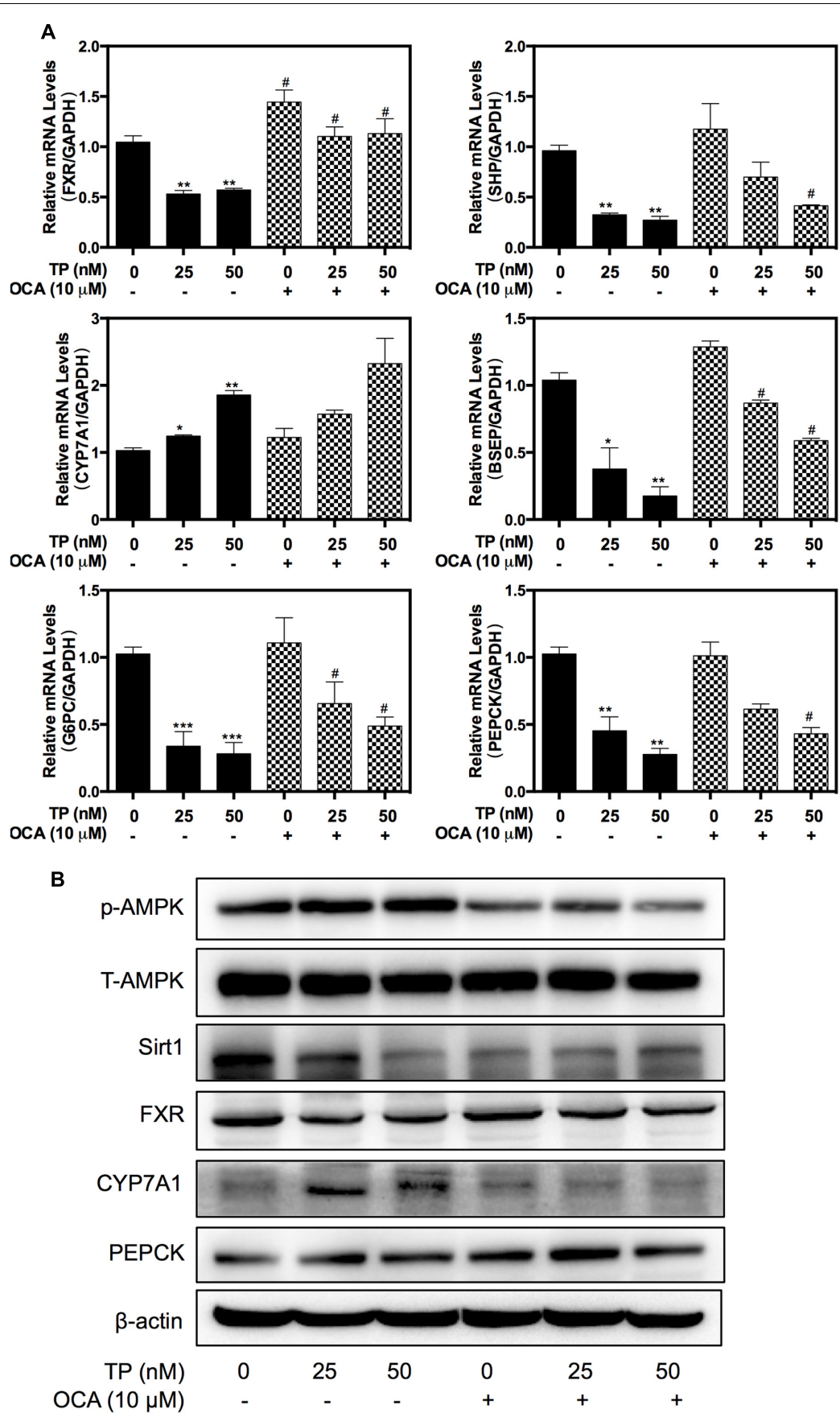

FIGURE 4 | Role of FXR in TP-induced hepatotoxicity. Primary rat hepatocytes were pretreated with the FXR agonist, obeticholic acid (OCA) (10 $\mu$ M), for one hour and then treated with TP for $8 \mathrm{~h}$. (A) The mRNA levels of FXR-target genes were determined using real-time RT-PCR and normalized to GAPDH as an internal control. (B) The target protein levels were determined using western blot analysis and normalized to $\beta$-actin as a loading control. Statistical significance relative to vehicle control, ${ }^{*} p<0.05,{ }^{* *} p<0.01,{ }^{* * *} p<0.001$; and statistical significance relative to the corresponding treated groups without $0 \mathrm{CA}$, ${ }^{\#} p<0.05$, ${ }^{\# \#} p<0.01$, $\# \#$ \#\# 0.001 .

Sirt 1 expression. For the data showed in Figures 3, 4, OCA may have stronger effects on FXR signaling pathway. The above data indicate that the loss of hepatic FXR was responsible for TPinduced disorders of the bile acid and glucose metabolism and the subsequent hepatotoxicity.

\section{Protective Effect of Sirt1 on TP-Induced Hepatotoxicity in Rats}

To investigate whether FXR activation would protect against TPinduced hepatotoxicity in vivo, we established an animal model of liver injury by oral administration of TP for 4 weeks and 
evaluated the protective effects of SRT1720 and OCA on TPinduced hepatotoxicity. Body weight was decreased, but not significantly different in the TP-treated group compared to the control group. However, as shown in Figure 5A, TP markedly increased the total bile acids (TBAs) and ALP levels in the serum, indicating cholestatic liver injury. Co-administration of OCA or SRT1720 reduced TP-induced hepatic injury. In addition, the serum glucose and total protein levels were decreased in the TP-treated group compared to the control group, but these effects were prevented in the OCA+TP and SRT1720+TP groups. The hepatic histopathological analysis revealed extensive hepatic parenchymal necrosis, vacuolated congestion and bile duct proliferation in the group treated with TP alone and these effects were significantly decreased when TP was combined with either OCA or SRT1720 (Figures 5B,C and Supplementary Figure S1).

Western blot analysis further indicated that TP co-treatment with either OCA or SRT1720 remarkably inhibited the TPinduced FXR suppression in the rat model (Figure 6A). OCA suppressed the AMPK phosphorylation and activated the FXR pathway, but SRT1720 did not affect the AMPK activation. The protein expression of specific FXR-targets including SHP and PEPCK was increased by the OCA or SRT1720 cotreatment. The CYP7A1 expression that had been increased by TP was suppressed by co-treatment with OCA or SRT1720. Similar to the results in the primary rat hepatocytes, OCA and SRT1720 also inhibited the TP-induced FXR suppression and the expression of key genes involved in bile acid synthesis and transport as well as hepatic gluconeogenesis at their mRNA levels (Figure 6B).

Taken together, these findings indicated that co-treatment with OCA or SRT1720 provided protection against TP-induced hepatotoxicity and supported our hypothesis that FXR activation plays a critical role in relieving TP-induced hepatotoxicity.

\section{DISCUSSION}

Drug-induced liver injury is the most frequent key cause for termination of drug development during or after preclinical studies and for drug withdrawal from the market (Schadt et al., 2016). Despite exhibiting excellent immunosuppressive and anti-tumor activities, $\mathrm{TP}$ has been greatly restricted in clinical application on the basis of a severe and cumulative hepatotoxicity. The toxicity of TP is closely related to its dose and duration. It has been reported that TP-induced hepatotoxicity occurred frequently in cases of either a single high dose or longterm low doses. Based on previous studies, a $400 \mu \mathrm{g} / \mathrm{kg}$ dose of TP administered orally for 28 days was used as the model for the evaluation of TP-induced toxicity in this study (Li et al., 2014). In addition, our recent studies indicated that TP induced more severe injury in female rats (Liu et al., 2015; Jiang et al., 2016). Similar to the results reported in the literature, TP increased serum total bile acid and ALP levels as compared to the control group in Wistar rats, indicating that TP by oral administration for 28 days significantly induced hepatotoxicity. However, the cellular mechanisms underlying TP-induced hepatotoxicity
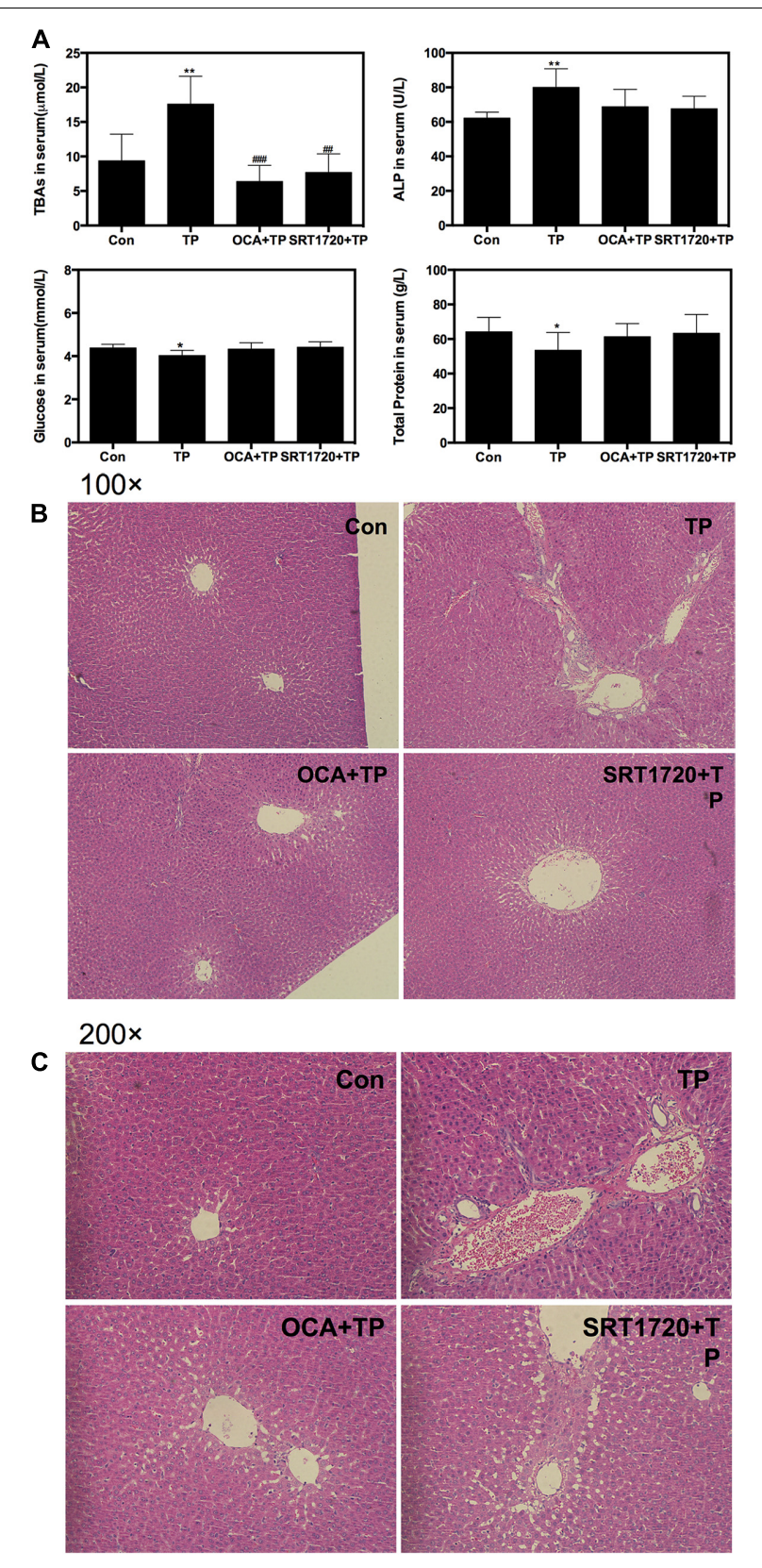

FIGURE 5 | Protective effect of Sirt1 on TP-induced hepatotoxicity in rat model. Female Wistar rats were treated as previously described in the Materials and Methods. (A) The serum levels of TBA, ALP, glucose and total protein levels were measured. $(\mathbf{B}, \mathbf{C})$ Representative images of the HE staining of the rat liver sections. The images were recorded using an Olympus microscope equipped with a $100 \times$ lens and $200 \times$ lens using an image recorder.

remain unclear. Our previous genome-wide microarray study indicated that TP alters the expression of 3329 genes that are involved in glucose metabolism and cellular stress pathways (Wang et al., 2013). In this study, we found that TP inhibited the Sirt1/FXR signaling pathway and caused dysregulation of bile acid homeostasis and hepatic gluconeogenesis (Figure 7). 


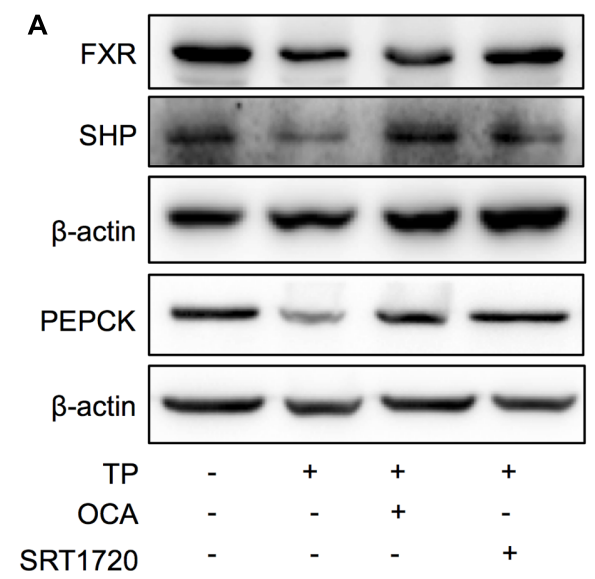

B
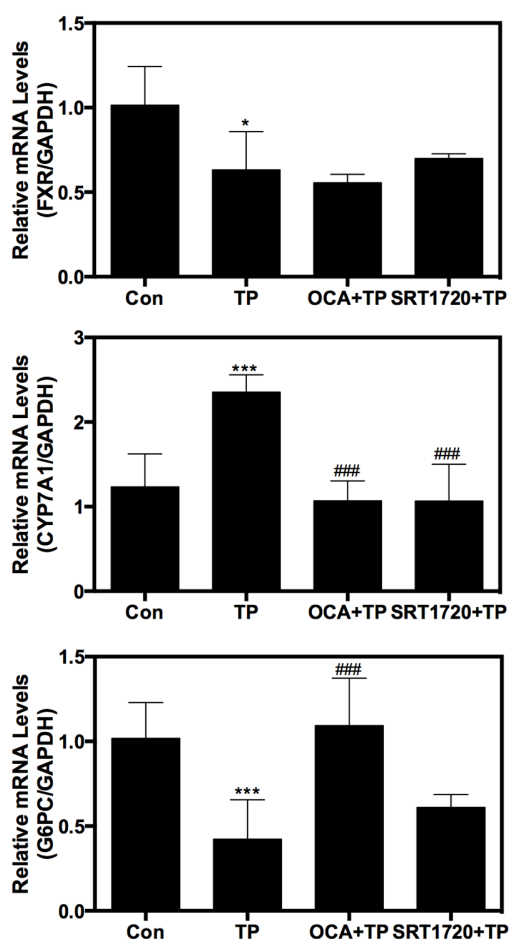
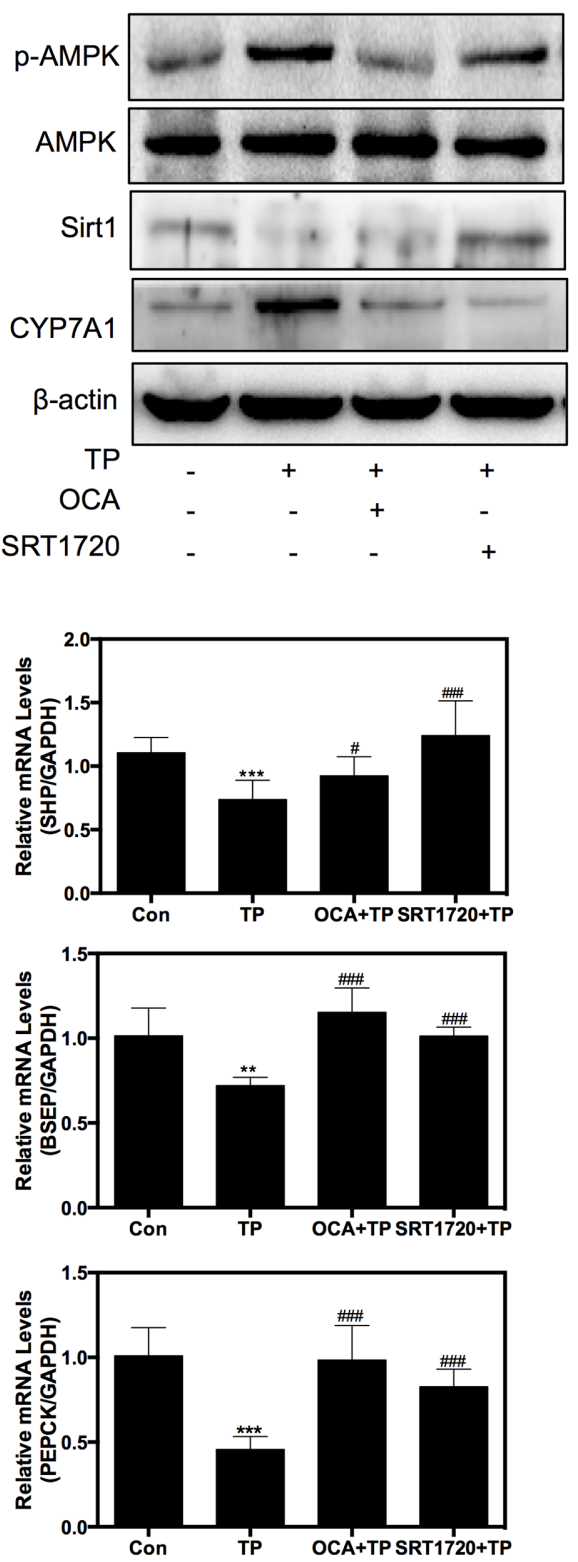

FIGURE 6 | Regulation of the expression of proteins and genes involved in TP-induced hepatotoxicity by SRT1720. Female Wistar rats were treated as previously described in the Section "Materials and Methods". (A) Representative immunoblots against p-AMPK, T-AMPK, Sirt1, FXR, and FXR-targets in the liver lysates are shown and normalized to $\beta$-actin as a loading control. (B) The relative mRNA levels of the relevant genes were determined using real-time RT-PCR and normalized to GAPDH as an internal control. Statistical significance relative to vehicle control, ${ }^{*} p<0.05,{ }^{* *} p<0.01$, ${ }^{* * *} p<0.001$; and statistical significance relative to the TP treated group, ${ }^{\#} p<0.05,{ }^{\# \#} p<0.01$, ${ }^{\# \# \# p<0.001 .}$

Our results indicate that inactivation of FXR may contribute to TP-induced hepatotoxicity. Furthermore, activation of Sirt1 through up-regulating FXR signaling pathway could have a protective effect against TP-induced disruption of hepatic metabolism.

Sirtl is a critical regulatory factor in the hepatic glucose metabolic processes. Hepatic deletion of Sirt1 leads to mild hypoglycemia, increased glucose tolerance, decreased hepatic glucose production, decreased serum cholesterol and increased hepatic content of free fatty acids and cholesterol, all of which can be reversed by Sirt1 overexpression (Rodgers and Puigserver, 2007). The current study showed that the rats treated with TP had mild hypoglycemia, which was correlated with changes of the protein expression levels involved in hepatic gluconeogenesis (Figure 6). Importantly, many of these changes were reversed by SRT1720. On the other hand, hepatic deletion of Sirt1 leads to dysfunctions in bile acid metabolism through downregulation of FXR signaling and the development of cholesterol gallstones on a lithogenic diet (Purushotham et al., 2012). Similarly, TP caused the accumulation of hepatic bile acids 


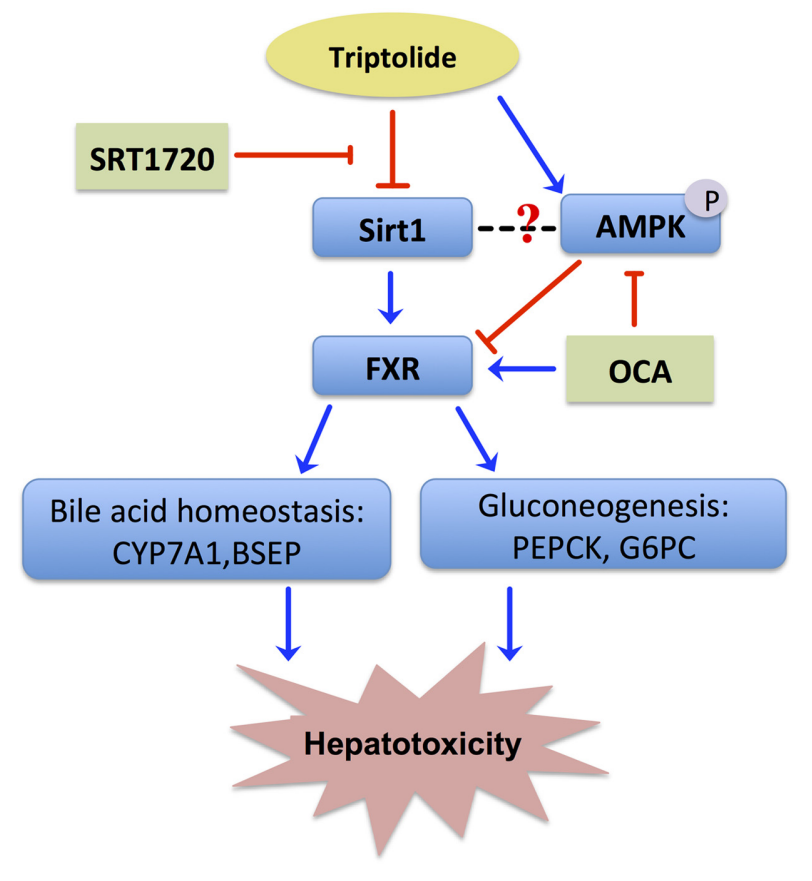

FIGURE 7 | Proposed signaling pathways involved in TP-induced hepatotoxicity. TP suppressed the FXR/SHP pathway via inhibiting the Sirt1 activity, which led to dysregulation of the expression of FXR-targeted genes involved in bile acid homeostasis and gluconeogenesis. Both Sirt1 agonist, SRT1720, and FXR agonist, OCA, reduced TP-induced disruption of hepatic metabolism and liver injury.

and increased serum ALP, which was blocked by SRT1720 (Figure 5).

FXR plays a crucial role in hepatic bile acid and glucose metabolism, hepatic regeneration, and the stress response to hepatotoxins and is frequently decreased in liver damage (Cariou, 2008; Lee F.Y. et al., 2010; Vaquero et al., 2013). FXR activation by agonists may represent an attractive therapeutic concept in cholestasis. GW4064 is a selective, non-steroidal FXR agonist (EC50 $=65 \mathrm{nM}$ ), while OCA is a potent and selective FXR agonist $($ EC50 $=99 \mathrm{nM})$. Now, OCA is used as a drug to treat primary biliary cholangitis, and is undergoing development for several other liver diseases and related disorders. This current study further demonstrated that dysfunction of FXR plays a key role in TP-induced hepatotoxicity. TP inhibited FXR expression and activity by down-regulating or inactivating hepatic Sirt1, resulting in disruption of bile acid and glucose metabolism and hepatic damage. It has been well-characterized that hepatic Sirt1 can modulate the FXR signaling pathway via different mechanisms. First, Sirt1 directly regulates the deacetylation of FXR, promotes its stability, inhibits its DNA-binding ability and transactivation activity (Kemper et al., 2009). Second, hepatic SIRT1 indirectly modulates FXR activity through HNF1 $\alpha$. In a liver-specific Sirt1 knockout mouse model, Sirt1 modulates the FXR signaling by down-regulating the HNF1 $\alpha$ recruitment to the FXR promoter and the expression of FXR (Purushotham et al., 2012). Third, hepatic Sirt1 may also indirectly increase the
mRNA levels of FXR by activating PGC-1 $\alpha$ (Zhang et al., 2004). Hepatic deletion of Sirt1 decreased co-activation of PGC-1 $\alpha$ (Purushotham et al., 2009). Finally, hepatic Sirt1 may indirectly regulate the transactivation activity of FXR by stimulating the biosynthesis of bile acids. The rate-limiting enzyme for bile acid synthesis, CYP7A1, is involved in cholesterol efflux and degradation, which is down-regulated by Sirt1 shRNA and induced by Sirt1 overexpression in the fasted state (Rodgers and Puigserver, 2007). In contrast, several lines of evidence suggest that the FXR-SHP pathway lies upstream of Sirt1 and regulates this protein via the $\mathrm{p} 53 / \mathrm{miR}-34 \mathrm{a}$ pathway (Lee J. et al., 2010). Therefore, Sirtl and the FXR signaling pathway mutually interact at multiple levels in response to various stimuli to coordinately regulate hepatic bile acid, cholesterol and glucose homeostasis. In this study, we found that TPinduced down-regulation of FXR protein expression was also blocked by SRT1720 (Figure 2). We will further explore the exact mechanism by which TP modulates the Sirt1 and FXR signaling pathway.

AMP-activated protein kinase is another important player in regulating hepatic metabolism. In this study, we found that SRT1720 had no effect on p-AMPK/AMPK. These results suggest either that AMPK is located upstream of Sirt1 or that the AMPKmediated regulation of FXR is independent of Sirt1 (Figure 2). Previous studies reported that activation of AMPK increases the NAD+ levels, which leads to activation of Sirt1 and deacetylation of its down-stream targets such as PGC-1 $\alpha$ (Chau et al., 2010; Kim et al., 2013), FOXO1 (Canto et al., 2009), FXR (GarciaRodriguez et al., 2014), and PPAR $\alpha$ (Kang et al., 2016). However, the current study indicated that TP simultaneously activated the phosphorylation of AMPK and suppressed the Sirt1 expression. Several studies have clearly demonstrated that AMPK negatively regulates Sirt1 expression or has no effect on Sirtlexpression ( $\mathrm{Zu}$ et al., 2010; Lee et al., 2012; Gollavilli et al., 2015). Lien reported that metformin induces activation of AMPK, which directly phosphorylates and regulates the FXR transcriptional activity and subsequently contributes to hepatocyte injury (Lien et al., 2014). Our recent study also showed that $17 \alpha$-ethinylestradiol (EE)induced activation and nuclear translocation of AMPK $\alpha 1$ plays a critical role in EE-induced cholestasis via down-regulation of the expression of FXR and bile acid receptors (Li et al., 2016). In the current study, we also found that rapidly and dose-dependently induced AMPK activation (Figure 4B). A semi-synthetic bile acid analog, OCA, not only activates FXR and improves bile acid and glucose metabolism, but also inhibits AMPK activation. OCA significantly inhibited TP-induced hepatotoxicity (Figures 4, 6). These findings further confirmed that AMPK also plays an important role in the TP-induced down-regulation of FXR. We will further evaluate whether activation of AMPK had any effect on the Sirtlexpression in TP-induced hepatotoxicity in the rat model.

\section{CONCLUSION}

Sirt1 is an endogenous activator of FXR in hepatocytes and often is decreased in liver injury. The present study demonstrated that 
TP-induced down-regulation of hepatic Sirt1 contributed to the reduced hepatic FXR levels and subsequently led to disruption of hepatic bile acid and glucose metabolism. Moreover, this Sirt1-FXR axis represents an important signaling pathway that may have the potential to ameliorate DILI in humans.

\section{AUTHOR CONTRIBUTIONS}

JY, HZ, LZ, and ZJ designed the experiments. JY, LS, LW, and XW performed the experiments. JY, TW, PH, HZ, and ZJ analyzed the data. JY, HH, HZ, LZ, and ZJ wrote the paper. All authors contributed to the editing of the paper and to scientific discussions.

\section{ACKNOWLEDGMENTS}

This study was supported by National Natural Science Foundation of China $(81320108029,81573690,81573514)$, the

\section{REFERENCES}

Banerjee, S., and Saluja, A. (2015). Minnelide, a novel drug for pancreatic and liver cancer. Pancreatology 15(Suppl. 4), S39-S43. doi: 10.1016/j.pan.2015. 05.472

Canto, C., Gerhart-Hines, Z., Feige, J. N., Lagouge, M., Noriega, L., Milne, J. C., et al. (2009). AMPK regulates energy expenditure by modulating NAD+ metabolism and SIRT1 activity. Nature 458, 1056-1060. doi: 10.1038/ nature 07813

Cao, R., Cronk, Z. X., Zha, W., Sun, L., Wang, X., Fang, Y., et al. (2010). Bile acids regulate hepatic gluconeogenic genes and farnesoid $X$ receptor via $G(a l p h a) i-$ protein-coupled receptors and the AKT pathway. J. Lipid Res. 51, 2234-2244. doi: 10.1194/jlr.M004929

Cariou, B. (2008). The farnesoid X receptor (FXR) as a new target in nonalcoholic steatohepatitis. Diabetes Metab. 34(6 Pt 2), 685-691. doi: 10.1016/ S1262-3636(08)74605-6

Chau, M. D., Gao, J., Yang, Q., Wu, Z., and Gromada, J. (2010). Fibroblast growth factor 21 regulates energy metabolism by activating the AMPK-SIRT1-PGC1alpha pathway. Proc. Natl. Acad. Sci. U.S.A. 107, 12553-12558. doi: 10.1073/ pnas. 1006962107

Chen, H., Huang, X., Min, J., Li, W., Zhang, R., Zhao, W., et al. (2016). Geniposidic acid protected against ANIT-induced hepatotoxity and acute intrahepatic cholestasis, due to Fxr-mediated regulation of Bsep and Mrp2. J. Ethnopharmacol. 179, 197-207. doi: 10.1016/j.jep.2015. 12.033

Fu, Q., Huang, X., Shu, B., Xue, M., Zhang, P., Wang, T., et al. (2011). Inhibition of mitochondrial respiratory chain is involved in triptolide-induced liver injury. Fitoterapia 82, 1241-1248. doi: 10.1016/j.fitote.2011.08.019

$\mathrm{Fu}$, Q., Jiang, Z. Z., and Zhang, L. Y. (2013). Impairment of triptolide on liver mitochondria in isolated liver mitochondria and HL7702 cell line. Chin. J. Integr. Med. 19, 683-688. doi: 10.1007/s11655-012-1265-x

Garcia-Rodriguez, J. L., Barbier-Torres, L., Fernandez-Alvarez, S., Gutierrez-de Juan, V., Monte, M. J., Halilbasic, E., et al. (2014). SIRT1 controls liver regeneration by regulating bile acid metabolism through farnesoid X receptor and mammalian target of rapamycin signaling. Hepatology 59, 1972-1983. doi: 10.1002/hep.26971

Gollavilli, P. N., Kanugula, A. K., Koyyada, R., Karnewar, S., Neeli, P. K., and Kotamraju, S. (2015). AMPK inhibits MTDH expression via GSK3beta and SIRT1 activation: potential role in triple negative breast cancer cell proliferation. FEBS J. 282, 3971-3985. doi: 10.1111/febs.13391

Jiang, Z., Huang, X., Huang, S., Guo, H., Wang, L., Li, X., et al. (2016). Sex-related differences of lipid metabolism induced by triptolide: the possible role of the
Natural Science Foundation of Jiangsu Province (BK20151439), the National "Major Scientific and Technological Special Project for Significant New Drugs" project (2015ZX09501004-002-004), Specific Fund for Public Interest Research of Traditional Chinese Medicine, Ministry of finance (201507004-002); National Institutes of Heath Grant R01 1R01DK104893 (to HZ and PH) and R01 DK-057543-11 (to PH and HZ), VA Merit Awards (to HZ; I01BX001390); VCU Massey Cancer Pilot grant (A35362 to $\mathrm{HZ})$.

\section{SUPPLEMENTARY MATERIAL}

The Supplementary Material for this article can be found online at: http://journal.frontiersin.org/article/10.3389/fphar. 2017.00260/full\#supplementary-material

FIGURE S1 | Triptolide (TP)-induced hepatic injury in rat model. Female Wistar rats were treated as previously described in the Materials and Methods. The images of the rat liver were recorded.

LXRalpha/SREBP-1 signaling pathway. Front. Pharmacol. 7:87. doi: 10.3389/ fphar.2016.00087

Jin, J., Sun, X., Zhao, Z., Wang, W., Qiu, Y., Fu, X., et al. (2015). Activation of the farnesoid $\mathrm{X}$ receptor attenuates triptolide-induced liver toxicity. Phytomedicine 22, 894-901. doi: 10.1016/j.phymed.2015.06.007

Kang, H. S., Cho, H. C., Lee, J. H., Oh, G. T., Koo, S. H., Park, B. H., et al. (2016). Metformin stimulates IGFBP-2 gene expression through PPARalpha in diabetic states. Sci. Rep. 6:23665. doi: 10.1038/srep23665

Kemper, J. K., Xiao, Z., Ponugoti, B., Miao, J., Fang, S., Kanamaluru, D., et al. (2009). FXR acetylation is normally dynamically regulated by p300 and SIRT1 but constitutively elevated in metabolic disease states. Cell Metab. 10, 392-404. doi: 10.1016/j.cmet.2009.09.009

Kim, M. Y., Lim, J. H., Youn, H. H., Hong, Y. A., Yang, K. S., Park, H. S., et al. (2013). Resveratrol prevents renal lipotoxicity and inhibits mesangial cell glucotoxicity in a manner dependent on the AMPK-SIRT1-PGC1alpha axis in $\mathrm{db} / \mathrm{db}$ mice. Diabetologia 56, 204-217. doi: 10.1007/s00125-012-2747-2

Lee, C. W., Wong, L. L., Tse, E. Y., Liu, H. F., Leong, V. Y., Lee, J. M., et al. (2012). AMPK promotes $\mathrm{p} 53$ acetylation via phosphorylation and inactivation of SIRT1 in liver cancer cells. Cancer Res. 72, 4394-4404. doi: 10.1158/0008-5472.CAN12-0429

Lee, F. Y., de Aguiar Vallim, T. Q., Chong, H. K., Zhang, Y., Liu, Y., Jones, S. A., et al. (2010). Activation of the farnesoid $\mathrm{X}$ receptor provides protection against acetaminophen-induced hepatic toxicity. Mol. Endocrinol. 24, 1626-1636. doi: 10.1210/me.2010-0117

Lee, J., Padhye, A., Sharma, A., Song, G., Miao, J., Mo, Y. Y., et al. (2010). A pathway involving farnesoid $\mathrm{X}$ receptor and small heterodimer partner positively regulates hepatic sirtuin 1 levels via microRNA-34a inhibition. J. Biol. Chem. 285, 12604-12611. doi: 10.1074/jbc.M109.094524

Li, X., Liu, R., Luo, L., Yu, L., Chen, X., Sun, L., et al. (2016). Role of AMP-activated protein kinase alphal in 17alpha-ethinylestradiol-induced cholestasis in rats. Arch. Toxicol. 91, 481-494. doi: 10.1007/s00204-016-1697-8

Li, X. J., Jiang, Z. Z., and Zhang, L. Y. (2014). Triptolide: progress on research in pharmacodynamics and toxicology. J. Ethnopharmacol. 155, 67-79. doi: 10.1016/j.jep.2014.06.006

Lien, F., Berthier, A., Bouchaert, E., Gheeraert, C., Alexandre, J., Porez, G., et al. (2014). Metformin interferes with bile acid homeostasis through AMPK-FXR crosstalk. J. Clin. Invest. 124, 1037-1051. doi: 10.1172/JCI68815

Liu, L., Zhang, J., Wang, Z., Xu, D., Jiang, Z., Wang, T., et al. (2015). Gender differences in the toxicokinetics of triptolide after single- and multiple-dose administration in rats. Drug Res. 65, 602-606. doi: 10.1055/s-0034-1395676

Liu, Y., Binz, J., Numerick, M. J., Dennis, S., Luo, G., Desai, B., et al. (2003). Hepatoprotection by the farnesoid X receptor agonist GW4064 in rat 
models of intra- and extrahepatic cholestasis. J. Clin. Invest. 112, 1678-1687. doi: 10.1172/JCI18945

Mudaliar, S., Henry, R. R., Sanyal, A. J., Morrow, L., Marschall, H. U., Kipnes, M., et al. (2013). Efficacy and safety of the farnesoid X receptor agonist obeticholic acid in patients with type 2 diabetes and nonalcoholic fatty liver disease. Gastroenterology 145, 574.e1-582.e1. doi: 10.1053/j.gastro.2013.05.042

Noel, O. F., Still, C. D., Argyropoulos, G., Edwards, M., and Gerhard, G. S. (2016). Bile acids, FXR, and metabolic effects of bariatric surgery. J. Obes. 2016:4390254. doi: $10.1155 / 2016 / 4390254$

Purushotham, A., Schug, T. T., Xu, Q., Surapureddi, S., Guo, X., and Li, X. (2009). Hepatocyte-specific deletion of SIRT1 alters fatty acid metabolism and results in hepatic steatosis and inflammation. Cell Metab. 9, 327-338. doi: 10.1016/j. cmet.2009.02.006

Purushotham, A., Xu, Q., Lu, J., Foley, J. F., Yan, X., Kim, D. H., et al. (2012). Hepatic deletion of SIRT1 decreases hepatocyte nuclear factor 1alpha/farnesoid $\mathrm{X}$ receptor signaling and induces formation of cholesterol gallstones in mice. Mol. Cell. Biol. 32, 1226-1236. doi: 10.1128/MCB.05988-11

Rivard, C., Geller, M., Schnettler, E., Saluja, M., Vogel, R. I., Saluja, A., et al. (2014). Inhibition of epithelial ovarian cancer by Minnelide, a water-soluble pro-drug. Gynecol. Oncol. 135, 318-324. doi: 10.1016/j.ygyno.2014.08.031

Rodgers, J. T., and Puigserver, P. (2007). Fasting-dependent glucose and lipid metabolic response through hepatic sirtuin 1. Proc. Natl. Acad. Sci. U.S.A. 104, 12861-12866. doi: 10.1073/pnas.0702509104

Schadt, H. S., Wolf, A., Pognan, F., Chibout, S. D., Merz, M., and Kullak-Ublick, G. A. (2016). Bile acids in drug induced liver injury: key players and surrogate markers. Clin. Res. Hepatol. Gastroenterol. 40, 257-266. doi: 10.1016/j.clinre. 2015.12.017

Stayrook, K. R., Bramlett, K. S., Savkur, R. S., Ficorilli, J., Cook, T., Christe, M. E., et al. (2005). Regulation of carbohydrate metabolism by the farnesoid X receptor. Endocrinology 146, 984-991. doi: 10.1210/en.2004-0965

Studer, E., Zhou, X., Zhao, R., Wang, Y., Takabe, K., Nagahashi, M., et al. (2012). Conjugated bile acids activate the sphingosine-1-phosphate receptor 2 in primary rodent hepatocytes. Hepatology 55, 267-276. doi: 10.1002/hep. 24681

Vaquero, J., Briz, O., Herraez, E., Muntane, J., and Marin, J. J. (2013). Activation of the nuclear receptor FXR enhances hepatocyte chemoprotection and liver tumor chemoresistance against genotoxic compounds. Biochim. Biophys. Acta 1833, 2212-2219. doi: 10.1016/j.bbamcr.2013.05.006

Wang, J., Jiang, Z., Ji, J., Wang, X., Wang, T., Zhang, Y., et al. (2013). Gene expression profiling and pathway analysis of hepatotoxicity induced by triptolide in Wistar rats. Food Chem. Toxicol. 58, 495-505. doi: 10.1016/j.fct. 2013.04.039

Wang, X., Jiang, Z., Cao, W., Yuan, Z., Sun, L., and Zhang, L. (2014). Th17/Treg imbalance in triptolide-induced liver injury. Fitoterapia 93, 245-251. doi: 10.1016/j.fitote.2014.01.006

Watanabe, M., Houten, S. M., Mataki, C., Christoffolete, M. A., Kim, B. W., Sato, H., et al. (2006). Bile acids induce energy expenditure by promoting intracellular thyroid hormone activation. Nature 439, 484-489. doi: 10.1038/ nature 04330

Yao, J., Jiang, Z., Duan, W., Huang, J., Zhang, L., Hu, L., et al. (2008). Involvement of mitochondrial pathway in triptolide-induced cytotoxicity in human normal liver L-02 cells. Biol. Pharm. Bull. 31, 592-597. doi: 10.1248/bpb.31.592

Zhang, Y., Castellani, L. W., Sinal, C. J., Gonzalez, F. J., and Edwards, P. A. (2004). Peroxisome proliferator-activated receptor-gamma coactivator 1alpha (PGC1alpha) regulates triglyceride metabolism by activation of the nuclear receptor FXR. Genes Dev. 18, 157-169. doi: 10.1101/gad.1138104

Zhou, J., Zhu, Q., and Yang, X. (1999). Clinical observation of side effects of Tripterygium preparation. Zhongguo Zhong Xi Yi Jie He Za Zhi 19, 77-79.

Zhou, Z. L., Yang, Y. X., Ding, J., Li, Y. C., and Miao, Z. H. (2012). Triptolide: structural modifications, structure-activity relationships, bioactivities, clinical development and mechanisms. Nat. Prod. Rep. 29, 457-475. doi: 10.1039/ c2np00088a

Ziaei, S., and Halaby, R. (2016). Immunosuppressive, anti-inflammatory and anticancer properties of triptolide: a mini review. Avicenna J. Phytomed. 6, 149-164.

Zu, Y., Liu, L., Lee, M. Y., Xu, C., Liang, Y., Man, R. Y., et al. (2010). SIRT1 promotes proliferation and prevents senescence through targeting LKB1 in primary porcine aortic endothelial cells. Circ. Res. 106, 1384-1393. doi: 10.1161/ CIRCRESAHA.109.215483

Conflict of Interest Statement: The authors declare that the research was conducted in the absence of any commercial or financial relationships that could be construed as a potential conflict of interest.

Copyright (c) 2017 Yang, Sun, Wang, Hassan, Wang, Hylemon, Wang, Zhou, Zhang and Jiang. This is an open-access article distributed under the terms of the Creative Commons Attribution License (CC BY). The use, distribution or reproduction in other forums is permitted, provided the original author(s) or licensor are credited and that the original publication in this journal is cited, in accordance with accepted academic practice. No use, distribution or reproduction is permitted which does not comply with these terms. 